\title{
A Soft Version of the Knaster-Tarski Fixed point Theorem with Applications
}

\author{
Bahru Tsegaye Leyew ${ }^{a, b}$ and Mujahid Abbas $^{a}$ \\ ${ }^{a}$ Department of Mathematics and Applied Mathematics, University of Pretoria, Lynnwood \\ road, Pretoria 0002, South Africa. \\ ${ }^{b}$ Department of Mathematics, Addis Ababa University, College of Natural Sciences, Addis \\ Ababa 1176, Ethiopia. \\ E-mail addresses: tsegayebah@gmail.com, mujahid.abbas@up.ac.za
}

\begin{abstract}
In this paper first we define a partial order on a soft set $(F, A)$ and introduce some related concepts. Then using the concept of a soft mapping introduced by Babitha and Sunil [Comput. Math. Appl., 60 (7) (2010), 1840-1849], a soft version of KnasterTarski fixed point theorem is obtained. Some examples are presented to support the concepts introduced and the results proved herein. As an application of our result, we show that the soft Knaster-Tarski fixed point theorem ensures the existence of a soft common fixed point for a commuting family of order-preserving soft mappings.
\end{abstract}

Keywords: Soft set, soft complete lattice, soft Knaster-Tarski fixed point, soft order preserving, soft least (soft greatest) fixed point.

2010 Mathematics Subject Classification: 47H10, 47H04, 47H07.

\section{Introduction and preliminaries}

Given a nonempty set $X$ and a function $f: X \rightarrow X, x^{*} \in X$ is a fixed point of $f$ if it remains invariant under the action of $f$, that is, $f x^{*}=x^{*}$. A relation $\leq$ on $X$ is called: $(i)$ reflexive if $x \leq x$ for all $x \in X$. (ii) transitive if $x \leq y$ and $y \leq z$ imply $x \leq z$ for all $x, y, z \in X$. (iii) antisymmetric if $x \leq y$ and $y \leq x$ imply $x=y$ for all $x, y \in X$. (iv) preorder if it is reflexive and transitive. A preorder is called partial order if it is antisymmetric. A partial order under which every pair of elements is comparable is called a total order or linear order; a totally ordered set is also called a chain (e.g., the natural numbers with their standard order). A partially ordered set (poset) $X$ is called a lattice if every subset of $X$ consisting of exactly two elements has a least upper bound and a greatest lower bound in $X$. A poset $X$ is complete lattice if every nonempty subset of $X$ has a least upper bound and a greatest lower bound in $X$.

Many existence problems in economics such as competitive equilibrium problems in general equilibrium theory, optimization problems, saddle point problems, Nash equilibrium 
problems in game theory, variational inequality problems and complementarity problems can be formulated as fixed point problems (see for instance $[6,7,8,9,11]$ and the references therein). The results providing sufficient conditions for existence, uniqueness and approximation of fixed point of certain mappings play significant role in game theory and optimization theory. One of the significant branch of fixed point theory is order-oriented fixed point theory which is studied in the environment created by the class of posets along with appropriate mappings satisfying certain conditions like order-preserving, expansivity, or various forms of order-continuity.

In 1907, Zermelo proved the following theorem.

Theorem 1.1 [20] Let $X$ be a poset in which each chain ( totally ordered set) has a least upper bound. If $f: X \rightarrow X$ satisfies $x \leq f x$ for each $x$ in $X$ (this condition is termed as expansivity), then there exists some $x$ in $X$ such that $f x=x$.

For a poset $(X, \leq)$, a mapping $f: X \rightarrow X$ is called an isotone mapping (or orderpreserving or monotone increasing) if $\forall x, y \in X: x \leq y$ implies that $f x \leq f y$. Similarly, $f$ is monotone decreasing (or order-reversing) if $\forall x, y \in X: x \leq y$ implies that $f y \leq f x$. All results in the rest of this paper are stated for order-preserving functions. Dual results exist for order-reversing functions, but we shall not mention them here.

In the mathematical areas of order and lattice theory, the Knaster-Tarski theorem, named after Bronistaw Knaster [13] and Alfred Tarski [18], states the following:

Theorem 1.2 Let $X$ be a complete lattice and $f: X \rightarrow X$ an isotone mapping. Then $f$ has a fixed point and the set of fixed points of $f$ in $X$ is also a complete lattice under the ordering induced by the underlying order.

It was Tarski [18] who stated the result in its most general form, and so the theorem is often known as Tarski's fixed point theorem. Some time earlier, Knaster and Tarski established the result for the special case where $X$ is the lattice of subsets of a set, the power set lattice [13]. Note that Banach contraction principle imposes a strong continuity condition on the mapping whereas the Knaster-Tarski fixed point theorem requires that the mapping is order-preserving but not necessarily continuous. The poset $(X, \leq)$ has the fixed point property if every order-preserving mapping of $(X, \leq)$ into itself has a fixed point. In 1995, Davis [10] proved that every lattice with the fixed point property is complete.

In 2009, Reem and Reich [17] established the existence of a diagram of 2 sites in any $m$ space and the existence of a double zone diagram (a fixed point of the second iteration Dom) of any number (possibly infinite) of sites based on the Knaster-Tarski fixed point theorem for order-preserving.

Real world problems are embedded with uncertainties. Molodtsov [15] introduced soft sets as a mathematical tool to handle uncertainty associated with real world data based problems. The contribution made by probability theory, fuzzy set theory, vague sets, rough sets and interval mathematics to deal with uncertainty is of vital importance but the problem of inadequacy of parameters has been successfully resolved by soft set theory. Soft set theory aims to provide enough tools to deal with uncertainty in a data and to represent it in a useful way. The distinguishing attribute of soft set theory is that unlike probability and fuzzy set theory, it does not uphold a precise quantity. 
Now this theory has become full-fledged research area and a vast amount of mathematical activity has been carried out to obtain many remarkable results showing the applicability of soft set theory in decision making, demand analysis, forecasting, information science, mathematics and other disciplines.

The study of soft set operations is vital for mathematicians and computer scientists to develop the theory of soft topological spaces. Maji et al. [14] introduced some basic algebraic operations on soft sets. Abbas et al. [3] proposed tolerance or dependence relation on the collection of soft sets and soft lattice structure. The concept of soft set relations as a soft subset of the Cartesian product of soft sets was introduced by Babitha and Sunil [4]. Employing the concept of a soft relation, they defined soft set function and studied some basic related properties.

Wardowski [19] obtained a soft fixed point results of a soft mapping in soft compact Hausdorff topological spaces. He also studied the properties of soft compact topological spaces. His main result is based on the fact that a decreasing sequence of nonempty soft closed subsets in soft compact topological spaces has a nonempty intersection.

Abbas et al. [1] gave a notion of soft contraction mappings based on the concept of soft elements of soft metric spaces and then obtained among other results, a theorem of Banach contraction principle type called soft contraction theorem in the setup of soft complete metric spaces. Recently, Abbas et al. [2], proved that a soft metric induces a compatible metric on the collection of all soft points of the absolute soft set provided that the set of parameter is a finite set. On the other hand, Stouti [16] gave a fuzzy version of Tariski's fixed point theorem for fuzzy monotone mappings on nonempty fuzzy complete lattice.

The aim of this paper is to initiate the study of soft fixed point theory in the framework of soft complete lattices and prove the soft version of the Kanster-Tarski fixed point theorem which would help in establishing a bridge between order oriented fixed point theory and soft fixed point theory.

Let us recall some basic definitions and known results in soft set theory, needed in the sequel.

Throughout this paper, $U$ refers to an initial universe, and $E$ the set of parameters for $U$. Denote by $P(U)$ the family of all subsets of $U$.

Definition 1.3 [15] If $F$ is a set valued mapping on $A \subseteq E$ taking values in $P(U)$, then a pair $(F, A)$ is called a soft set over $U$.

A soft set $(F, A)$ can be seen as a parametrized family of subsets of the set $U$. For each $e$ in $A$, the set $F(e)$ in $U$ is called $e$-approximate element of the soft set $(F, A)$.

Thus a soft set $(F, A)$ is identified with the set $\{(e, F(e)): e \in A\}$. We call $(e, F(e)) \tilde{\in}(F, A)$ the soft ingredient of the soft set $(F, A)$.

Two soft ingredients $(e, F(e))$ and $\left(e^{\prime}, F\left(e^{\prime}\right)\right)$ in $(F, A)$ are said to be equal if and only if $e=e^{\prime}$ and $F(e)=F\left(e^{\prime}\right)$. If $F(e)=\emptyset$ for $e \in A$, we say $(e, F(e))$ empty soft ingredient of the soft set $(F, A)$.

Definition 1.4 [14] A soft set $(F, A)$ over $U$ is said to be an absolute soft set, denoted by $\tilde{U}$, if $F(e)=U$ for all $e \in A$. 
Definition 1.5 [14] A soft set $(F, A)$ over $U$ is said to be a null soft set over $U$ if $F(e)=\emptyset$ for all $e \in A$.

Definition 1.6 [14] Let $(F, A)$ and $(G, B)$ be two soft sets over a common universe $U$. We say that $(F, A)$ is a soft subset of $(G, B)$ or $(G, B)$ is a soft superset of $(F, A)$ if $A \subseteq B$ and $F(e) \subseteq G(e)$ for all $e \in A$. We denote it as $(F, A) \tilde{\subseteq}(G, B)$. Two soft sets $(F, A)$ and $(G, B)$ over a common universe $U$ are said to be soft equal if $(F, A) \tilde{\subseteq}(G, B)$ and $(G, B) \tilde{\subseteq}(F, A)$.

Recently, Irfan et al. [12] noticed that if the set of parameters is a lattice, then a monotone or an isotone mapping give rise to notions of lattice ordered soft sets and anti-lattice ordered soft sets, respectively. Motivated by Definition 16 in [12], we define a soft relation, which we will show is a partial order, on the soft set $(F, A)$ as follows:

Definition 1.7 Let $(F, A)$ be a soft set and $\leq_{p}$ a partial order on $A$. In $(F, A)$, we define a soft relation $\leq_{s}$ in the following way:

$$
(x, F(x)) \leq_{s}(y, F(y)) \text { if and only if } x \leq_{p} y \text { and } F(x) \subseteq F(y)
$$

for $x, y \in A$.

It is now straightforward to check that:

(i) $\leq_{s}$ is reflexive, that is, $(x, F(x)) \leq_{s}(x, F(x))$ for all $x$ in $A$.

(ii) $\leq_{s}$ is antisymmetric, that is, for any $x, y \in A,(x, F(x)) \leq_{s}(y, F(y))$ and $(y, F(y)) \leq_{s}$ $(x, F(x))$ imply that $(x, F(x))=(y, F(y))$.

(iii) $\leq_{s}$ is transitive, that is, $(x, F(x)) \leq_{s}(y, F(y))$ and $(y, F(y)) \leq_{s}(z, F(z))$ imply that $(x, F(x)) \leq_{s}(z, F(z))$ for any $x, y, z \in A$.

In this case, we say $(F, A)$ is partially ordered soft set or simply ordered soft set and we denote it by $\left((F, A), \leq_{s}\right)$.

Example 1.8 Let $U=\left\{r_{1}, r_{2}, r_{3}, r_{4}, r_{5}\right\}$ be a set of five class rooms (a universal set) and $A=\left\{a_{1}, a_{2}, a_{3}, a_{4}\right\}$ a set of parameters, where $a_{1}, a_{2}, a_{3}$ and $a_{4}$ stand for very small room, small room, big room, and very big room, respectively. Clearly, there is natural order among the parameters of set $A$ which can be described as: $a_{1} \leq_{p} a_{2} \leq_{p} a_{3} \leq_{p} a_{4}$. The soft sets $(F, A)$ and $(G, A)$ are given by

$$
\begin{aligned}
& (F, A)=\left\{\left(a_{1},\left\{r_{5}\right\}\right),\left(a_{2},\left\{r_{1}, r_{3}, r_{5}\right\}\right),\left(a_{3},\left\{r_{1}, r_{3}, r_{4}, r_{5}\right\}\right),\left(a_{4},\left\{r_{1}, r_{2}, r_{3}, r_{4}, r_{5}\right\}\right)\right\} \\
& (G, A)=\left\{\left(a_{1},\left\{r_{2}\right\}\right),\left(a_{2},\left\{r_{1}, r_{3}, r_{5}\right\}\right),\left(a_{3},\left\{r_{1}, r_{3}, r_{4}\right\}\right),\left(a_{4},\left\{r_{3}, r_{4}\right\}\right)\right\} .
\end{aligned}
$$

In this case, the soft sets $(F, A)$ and $(G, A)$ describe different types of class rooms. Obviously, $F\left(a_{1}\right) \subseteq F\left(a_{2}\right) \subseteq F\left(a_{3}\right) \subseteq F\left(a_{4}\right)$ and hence we have

$$
\left(a_{1}, F\left(a_{1}\right)\right) \leq_{s}\left(a_{2}, F\left(a_{2}\right)\right) \leq_{s}\left(a_{3}, F\left(a_{3}\right)\right) \leq_{s}\left(a_{4}, F\left(a_{4}\right)\right) .
$$

On the other hand, $(x, G(x)) \leq_{s}(y, G(y))$ does not hold for every $x, y \in A$ with $x \leq_{p} y$. Thus $(F, A)$ is an ordered soft set but $(G, A)$ is not. 
Let $\left((F, A), \leq_{s}\right)$ be an ordered soft set. We say that $(x, F(x))<_{s}(y, F(y))$ if and only if $\left((x, F(x)) \leq_{s}(y, F(y))\right.$ and either $x \neq y$ or $\left.F(x) \neq F(y)\right)$. We now define some related concepts based on the notion of a partial order on a soft set $(F, A)$.

Definition 1.9 The soft ingredients $(x, F(x))$ and $(y, F(y))$ of a partial ordered soft set $\left((F, A), \leq_{s}\right)$ are called comparable if and only if $(x, F(x)) \leq_{s}(y, F(y))$ or $(y, F(y)) \leq_{s}$ $(x, F(x))$ holds.

Definition 1.10 A soft subset $(G, B)$ of an ordered soft set $\left((F, A), \leq_{s}\right)$ is called a soft chain or totally ordered soft set in $(F, A)$ if every two soft ingredients of $(G, B)$ are comparable.

Definition 1.11 The partial order $\leq_{s}$ on a soft set $(F, A)$ is called total or linear ordering on $(F, A)$ if every two soft ingredients of $(F, A)$ are comparable.

Definition 1.12 The soft ingredient $(x, F(x))$ of a soft set $(F, A)$ ordered by $\leq_{s}$ is called:

(a) soft least ingredient of $(F, A)$ if $(x, F(x)) \leq_{s}(y, F(y))$ for all $(y, F(y)) \tilde{\in}(F, A)$.

(b) soft minimal ingredient of $(F, A)$ if there exists no soft ingredient $(y, F(y)) \tilde{\in}(F, A)$ such that $(y, F(y)) \leq_{s}(x, F(x))$ and $(y, F(y)) \neq(x, F(x))$.

(c) soft greatest ingredient of $(F, A)$ if $(y, F(y)) \leq_{s}(x, F(x))$ for all $(y, F(y)) \tilde{\in}(F, A)$.

(d) soft maximal ingredient of $(F, A)$ if there exists no soft ingredient $(y, F(y)) \tilde{\in}(F, A)$ such that $(x, F(x)) \leq_{s}(y, F(y))$ and $(x, F(x)) \neq(y, F(y))$.

A soft chain clearly has at most one soft maximal (soft minimal) ingredient.

Definition 1.13 Let $(G, B)$ be a soft subset of an ordered soft set $\left((F, A), \leq_{s}\right)$ and $x \in A$. The soft ingredient $(x, F(x)) \tilde{\in}(F, A)$ is called:

i) soft lower bound of $(G, B)$ in $\left((F, A), \leq_{s}\right)$ if $(x, F(x)) \leq_{s}(y, F(y))$ for all $(y, F(y)) \tilde{\in}(G, B)$.

ii) soft infimum or the soft greatest lower bound of $(G, B)$ in $\left((F, A), \leq_{s}\right)$ if it is the soft greatest ingredient of the soft set of all soft lower bounds of $(G, B)$ in $\left((F, A), \leq_{s}\right)$.

iii) soft upper bound of $(G, B)$ in $\left((F, A), \leq_{s}\right)$ if $(y, F(y)) \leq_{s}(x, F(x))$ for all $(y, F(y)) \tilde{\epsilon}(G, B)$.

iv) soft supremum or the soft least upper bound of $(G, B)$ in $\left((F, A), \leq_{s}\right)$ if it is the soft least ingredient of the soft set of all soft upper bounds of $(G, B)$ in $\left((F, A), \leq_{s}\right)$.

Definition 1.14 A soft set $(F, A)$ ordered by $\leq_{s}$ is a soft lattice if every soft subset $(G, B)$ of $(F, A)$ consisting of exactly two soft ingredients has the soft least upper bound and the soft greatest lower bound in $(F, A)$.

Definition 1.15 A soft set $(F, A)$ ordered by $\leq_{s}$ is a soft complete lattice if every soft subset $(G, B)$ of $(F, A)$ has soft least upper bound and soft greatest lower bound in $(F, A)$. 
To illustrate the above concepts, we give the following example.

Example 1.16 Suppose that $U=\left\{u_{1}, u_{2}, u_{3}, u_{4}, u_{5}, u_{6}\right\}$ is a given universe and $A=\left\{e_{1}, e_{2}, e_{3}, e_{5}, e_{6}\right\}$, and $B=\left\{e_{1}, e_{3}, e_{5}\right\}$ are subsets of a set $E=\left\{e_{1}, e_{2}, e_{3}, e_{4}, e_{5}, e_{6}\right\}$ of parameters. Soft sets $(F, A)$ and $(G, B)$ over the common universe $U$ are given as:

$$
(F, A)=\left\{\begin{array}{l}
\left(e_{1},\left\{u_{1}, u_{2}\right\}\right),\left(e_{2},\left\{u_{1}, u_{3}\right\}\right),\left(e_{3},\left\{u_{1}, u_{2}, u_{3}\right\}\right) \\
\left(e_{5},\left\{u_{1}, u_{2}, u_{3}, u_{4}\right\}\right),\left(e_{6},\left\{u_{1}, u_{2}, u_{3}, u_{4}, u_{6}\right\}\right)
\end{array}\right\}
$$

and

$$
(G, B)=\left\{\left(e_{1},\left\{u_{2}\right\}\right),\left(e_{3},\left\{u_{1}, u_{2}, u_{3}\right\}\right),\left(e_{5},\left\{u_{1}, u_{2}, u_{3}, u_{4}\right\}\right)\right\}
$$

where

$$
\begin{aligned}
& F\left(e_{1}\right)=\left\{u_{1}, u_{2}\right\}, F\left(e_{2}\right)=\left\{u_{1}, u_{3}\right\}, F\left(e_{3}\right)=\left\{u_{1}, u_{2}, u_{3}\right\} \\
& F\left(e_{5}\right)=\left\{u_{1}, u_{2}, u_{3}, u_{4}\right\} \text { and } F\left(e_{6}\right)=\left\{u_{1}, u_{2}, u_{3}, u_{4}, u_{6}\right\} \\
& G\left(e_{1}\right)=\left\{u_{2}\right\}, G\left(e_{3}\right)=\left\{u_{1}, u_{2}, u_{3}\right\} \text { and } G\left(e_{5}\right)=\left\{u_{1}, u_{2}, u_{3}, u_{4}\right\}
\end{aligned}
$$

\begin{tabular}{|l|l|l|l|l|l|l|}
\hline & $\mathrm{u}_{1}$ & $\mathrm{u}_{2}$ & $\mathrm{u}_{3}$ & $\mathrm{u}_{4}$ & $\mathrm{u}_{5}$ & $\mathrm{u}_{6}$ \\
\hline \hline $\mathrm{e}_{1}$ & 1 & 1 & 0 & 0 & 0 & 0 \\
\hline $\mathrm{e}_{2}$ & 1 & 0 & 1 & 0 & 0 & 0 \\
\hline $\mathrm{e}_{3}$ & 1 & 1 & 1 & 0 & 0 & 0 \\
\hline $\mathrm{e}_{5}$ & 1 & 1 & 1 & 1 & 0 & 0 \\
\hline $\mathrm{e}_{6}$ & 1 & 1 & 1 & 1 & 0 & 1 \\
\hline \hline
\end{tabular}

Table 1: Ordered soft set $(F, A)$

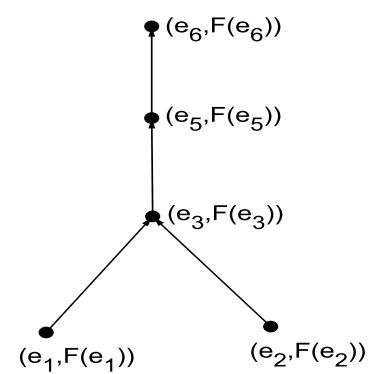

Figure 1: Order of ingredients of $(F, A)$

\begin{tabular}{|l|r|r|r|r|r|r|}
\hline & $\mathrm{u}_{1}$ & $\mathrm{u}_{2}$ & $\mathrm{u}_{3}$ & $\mathrm{u}_{4}$ & $\mathrm{u}_{5}$ & $\mathrm{u}_{6}$ \\
\hline \hline $\mathrm{e}_{1}$ & 0 & 1 & 0 & 0 & 0 & 0 \\
\hline $\mathrm{e}_{3}$ & 1 & 1 & 1 & 0 & 0 & 0 \\
\hline $\mathrm{e}_{5}$ & 1 & 1 & 1 & 1 & 0 & 0 \\
\hline \hline
\end{tabular}

Table 2: Ordered soft set $(G, B)$

In Figure 1 and Figure 2 the order among the ingredients of soft sets $(F, A)$ and $(G, B)$ are shown. For computer applications it is convenient to represent a soft set in tabular form. Table 1 and Table 2, represent the soft sets $(F, A)$ and $(G, B)$ respectively. If in set $U$, $u_{i} \in F\left(e_{j}\right)$ and $u_{i} \in G\left(e_{j}\right)$ we write 1 , otherwise 0 for each $i, j \in\{1,2,3,4,5,6\}$. From Table 1 , it is clear that $F\left(e_{1}\right) \subseteq F\left(e_{3}\right) \subseteq F\left(e_{5}\right) \subseteq F\left(e_{6}\right)$ and $F\left(e_{2}\right) \subseteq F\left(e_{3}\right) \subseteq F\left(e_{5}\right) \subseteq F\left(e_{6}\right)$. From Table 2, it is clear that $G\left(e_{1}\right) \subseteq G\left(e_{3}\right) \subseteq G\left(e_{5}\right)$. Obviously $(G, B)$ is the soft subset 
of $(F, A)$. Define the partial order $\leq_{p}$ on the set $A$ of parameters by $e_{i} \leq_{p} e_{j}$ if and only if $i \leq j$. Clearly, $\left((F, A), \leq_{S}\right)$ and $\left((G, B), \leq_{S}\right)$ are partially ordered soft sets. As soft ingredients $\left(e_{1}, F\left(e_{1}\right)\right)$ and $\left(e_{2}, F\left(e_{2}\right)\right)$ of the soft set $(F, A)$ are incomparable, therefore $(F, A)$ is not soft complete lattice. On the other hand, the soft set $(G, B)$ is a soft complete lattice. Moreover, $(G, B)$ is a soft chain in $\left((F, A), \leq_{S}\right)$. Indeed, every two soft ingredients of $(G, B)$ are comparable. Note that (a) the soft ingredients $\left(e_{1}, F\left(e_{1}\right)\right)$ and $\left(e_{2}, F\left(e_{2}\right)\right)$ of the soft set $(F, A)$ are soft minimal ingredients of $(F, A)$; $(b)$ the soft ingredient $\left(e_{6}, F\left(e_{6}\right)\right)$ of the soft set $(F, A)$ is the soft maximal ingredient and also the soft greatest ingredient of $(F, A)$; $(c)$ the soft ingredient $\left(e_{1}, G\left(e_{1}\right)\right)$ is the soft minimal ingredient and also the soft least ingredient of the soft set $(G, B)$; (d) the soft ingredient $\left(e_{5}, G\left(e_{5}\right)\right)$ is the soft maximal ingredient and also the soft greatest ingredient of the soft set $(G, B) ;(e)\left(e_{5}, F\left(e_{5}\right)\right)$ and $\left(e_{6}, F\left(e_{6}\right)\right)$ are soft upper bounds of $(G, B)$ in the ordered soft set $\left((F, A), \leq_{S}\right) ;(f)$ the soft ingredient $\left(e_{5}, F\left(e_{5}\right)\right)$ of the soft set $(F, A)$ is the soft supremum of $(G, B) ;(g)$ the soft ingredient $\left(e_{1}, F\left(e_{1}\right)\right)$ of the soft set $(F, A)$ is soft lower bound of $(G, B)$ and also the soft infimum of $(G, B)$ in $\left((F, A), \leq_{S}\right)$.

Suppose $(F, A)$ and $(G, B)$ are two soft sets over a common universe $U$. They may well have quite different partial orders but for sake of simplicity, we will use the same notation $\leq_{S}$ for each.

Motivated by the work of Babitha and Sunil [5], we get the following slight invariant of the concept of a soft mapping.

Definition 1.17 Let $(F, A)$ and $(G, B)$ be two soft sets over a common universe $U$. A soft relation

$$
f \tilde{\subseteq}(F, A) \tilde{\times}(G, B)=\{((x, y), F(x) \times G(y)):(x, F(x)) \tilde{\in}(F, A),(y, G(y)) \tilde{\in}(G, B)\}
$$

is called a soft mapping from $(F, A)$ to $(G, B)$, denoted by $f:(F, A) \stackrel{\sim}{\rightarrow}(G, B)$, if the following two conditions are satisfied:

(M1) for each soft ingredient $(x, F(x)) \tilde{\in}(F, A)$, there exists only one soft ingredient $(y, G(y)) \tilde{\in}(G, B)$ such that $((x, y), F(x) \times G(y)) \tilde{\in} f$. We write it as $(x, F(x)) f(y, G(y))$ or $f((x, F(x)))=$ $(y, G(y))$;

(M2) for each empty soft ingredient $(x, F(x)) \tilde{\in}(F, A), f((x, F(x)))$ is an empty soft ingredient of $(G, B)$.

Definition 1.18 Let $(F, A),(G, B)$ and $(H, C)$ be soft sets over a common universe $U$.

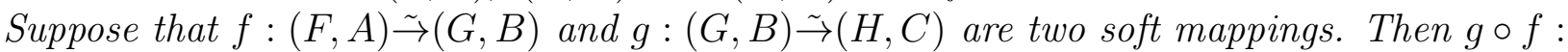
$(F, A) \stackrel{\sim}{\rightarrow}(H, C)$ is also a soft mapping defined by $g \circ f((x, F(x)))=g(f((x, F(x))))$ for all $(x, F(x)) \tilde{\epsilon}(F, A)$.

Definition 1.19 Let $(F, A)$ be a soft set ordered by $\leq_{s}$. A soft mapping $f:(F, A) \stackrel{\sim}{\rightarrow}(F, A)$ is called soft isotone or soft order-preserving if for any $(x, F(x)),(y, F(y)) \tilde{\in}(F, A)$,

$$
(x, F(x)) \leq_{S}(y, F(y)) \text { implies that } f((x, F(x))) \leq_{S} f((y, F(y))) .
$$


Example 1.20 Let $\left((F, A), \leq_{S}\right)$ and $\left((G, B), \leq_{S}\right)$ be partially ordered soft sets as given in Example 1.16. Define a soft relation $f:(F, A) \stackrel{\sim}{\rightarrow}(G, B)$ by

$$
f=\left\{\begin{array}{l}
\left(\left(e_{1}, e_{1}\right), F\left(e_{1}\right) \times G\left(e_{1}\right)\right),\left(\left(e_{2}, e_{3}\right), F\left(e_{2}\right) \times G\left(e_{3}\right)\right), \\
\left(\left(e_{3}, e_{3}\right), F\left(e_{3}\right) \times G\left(e_{3}\right)\right),\left(\left(e_{5}, e_{3}\right), F\left(e_{5}\right) \times G\left(e_{3}\right)\right), \\
\left(\left(e_{6}, e_{5}\right), F\left(e_{6}\right) \times G\left(e_{5}\right)\right)
\end{array}\right\} .
$$

That is,

$$
\begin{array}{ll}
f\left(\left(e_{1}, F\left(e_{1}\right)\right)\right)=\left(e_{1}, G\left(e_{1}\right)\right), & f\left(\left(e_{2}, F\left(e_{2}\right)\right)\right)=\left(e_{3}, G\left(e_{3}\right)\right), \quad f\left(\left(e_{3}, F\left(e_{3}\right)\right)\right)=\left(e_{3}, G\left(e_{3}\right)\right), \\
f\left(\left(e_{5}, F\left(e_{5}\right)\right)\right)=\left(e_{3}, G\left(e_{3}\right)\right), & f\left(\left(e_{6}, F\left(e_{6}\right)\right)\right)=\left(e_{5}, G\left(e_{5}\right)\right) .
\end{array}
$$

Clearly $f$ is a soft mapping from $(F, A)$ to $(G, B)$. Note that $f$ is a soft order-preserving mapping.

In Examples 1.16 and 1.20, we take $A=\left\{e_{1}, e_{2}, e_{3}, e_{4}, e_{5}, e_{6}\right\}$ and $B=\left\{e_{1}, e_{3}, e_{4}, e_{5}\right\}$ but $(F, A),(G, B)$ and $f$ remain the same. Note that $F\left(e_{4}\right)=\emptyset$ and $G\left(e_{4}\right)=\emptyset$. Hence $\left(e_{4}, F\left(e_{4}\right)\right)$ is an empty soft ingredient of the soft set $(F, A)$. Moreover, $f\left(\left(e_{4}, F\left(e_{4}\right)\right)\right)=\left(e_{4}, G\left(e_{4}\right)\right)$ is an empty soft ingredient of the soft set $(G, B)$.

Definition 1.21 Let $(F, A)$ be a soft set and $f:(F, A) \stackrel{\sim}{\rightarrow}(F, A)$ a soft mapping. A soft ingredient $\left(x^{*}, F\left(x^{*}\right)\right) \tilde{\epsilon}(F, A)$ is said to be a soft fixed point of $f$ if $\left(\left(x^{*}, x^{*}\right), F\left(x^{*}\right) \times F\left(x^{*}\right)\right) \tilde{\epsilon} f$, that is, $f\left(\left(x^{*}, F\left(x^{*}\right)\right)\right)=\left(x^{*}, F\left(x^{*}\right)\right)$. The soft fixed point set of $f$ in $(F, A)$ is a soft set of the form given by

$$
\left(F_{f}, A^{0}\right)=\{(x, F(x)) \tilde{\epsilon}(F, A): \quad((x, x), F(x) \times F(x)) \tilde{\epsilon} f\},
$$

where $A^{0}$ is the set of all parameters $x$ for which $(x, F(x)) \tilde{\epsilon}(F, A)$ and $((x, x), F(x) \times F(x)) \tilde{\epsilon} f$ hold.

Example 1.22 Let $\left((F, A), \leq_{S}\right)$ be partially ordered soft set as given in Example 1.16. Define a soft relation $f:(F, A) \stackrel{\sim}{\rightarrow}(F, A)$ by

$$
f=\left\{\begin{array}{l}
\left(\left(e_{1}, e_{1}\right), F\left(e_{1}\right) \times F\left(e_{1}\right)\right),\left(\left(e_{2}, e_{3}\right), F\left(e_{2}\right) \times F\left(e_{3}\right)\right), \\
\left(\left(e_{3}, e_{3}\right), F\left(e_{3}\right) \times F\left(e_{3}\right)\right),\left(\left(e_{5}, e_{3}\right), F\left(e_{5}\right) \times F\left(e_{3}\right)\right), \\
\left(\left(e_{6}, e_{5}\right), F\left(e_{6}\right) \times F\left(e_{5}\right)\right)
\end{array}\right\}
$$

That is,

$$
\begin{array}{ll}
f\left(\left(e_{1}, F\left(e_{1}\right)\right)\right)=\left(e_{1}, F\left(e_{1}\right)\right), & f\left(\left(e_{2}, F\left(e_{2}\right)\right)\right)=\left(e_{3}, F\left(e_{3}\right)\right), \quad f\left(\left(e_{3}, F\left(e_{3}\right)\right)\right)=\left(e_{3}, F\left(e_{3}\right)\right), \\
f\left(\left(e_{5}, F\left(e_{5}\right)\right)\right)=\left(e_{3}, F\left(e_{3}\right)\right), & f\left(\left(e_{6}, F\left(e_{6}\right)\right)\right)=\left(e_{5}, F\left(e_{5}\right)\right) .
\end{array}
$$

Clearly $f$ is a soft mapping from $(F, A)$ to $(F, A)$. Note that $f$ is a soft order-preserving mapping. The soft ingredients $\left(e_{1}, F\left(e_{1}\right)\right)$ and $\left(e_{3}, F\left(e_{3}\right)\right)$ are soft fixed points of $f$. Hence $\left(F_{f}, A^{0}\right)=\left\{\left(e_{1}, F\left(e_{1}\right)\right),\left(e_{3}, F\left(e_{3}\right)\right)\right\}$ is a soft fixed point subset of $(F, A)$.

Definition 1.23 Let $(F, A)$ be a soft set ordered by $\leq_{s}$ and $(a, F(a)),(b, F(b))$ two soft ingredients of $(F, A)$ satisfying $(a, F(a)) \leq_{S}(b, F(b))$. A soft interval in $(F, A)$ is a soft set of the form given by

$$
(R, B)=\left\{(x, F(x)) \tilde{\epsilon}(F, A):(a, F(a)) \leq_{S}(x, F(x)) \leq_{S}(b, F(b))\right\}
$$

where $B=\left\{x \in A: a \leq_{p} x \leq_{p} b\right\}$ is a subset of $A$ and $R(x)=F(x)$ whenever $x \in B$. 


\section{A soft Knaster-Tarski fixed point theorem and its applications}

In this section, we establish the existence of a soft fixed point of soft isotone mapping defined on a soft complete lattice, which is essentially the soft version of the well-known KnasterTarski fixed point theorem.

We start with the following lemma.

Lemma 2.1 Suppose that a soft ordered set $\left((F, A), \leq_{S}\right)$ is a soft complete lattice and $(a, F(a)),(b, F(b))$ are any two soft ingredients of $(F, A)$ such that $(a, F(a)) \leq_{s}(b, F(b))$. Then the soft interval is a soft complete lattice.

Proof. In (3), clearly, $(R, B)$ is a soft subset of $(F, A)$. Let $(S, C)$ be any soft subset of $(R, B)$. As $(F, A)$ is a soft complete lattice and $(S, C) \tilde{\subseteq}(F, A)$, the soft set $(S, C)$ has a soft least upper bound $\left(s^{*}, F\left(s^{*}\right)\right) \tilde{\in}(F, A)$. We now show that $\left(s^{*}, F\left(s^{*}\right)\right) \tilde{\in}(R, B)$. Note that $(b, F(b))$ is a soft upper bound for $(R, B)$. Since $(S, C) \tilde{\subseteq}(R, B)$, therefore the soft ingredient $(b, F(b))$ is soft upper bound of $(S, C)$ and hence $\left(s^{*}, F\left(s^{*}\right)\right) \leq_{s}(b, F(b))$. Also, the soft ingredient $(a, F(a))$ is a soft lower bound for $(R, B)$. As $(S, C) \tilde{\subseteq}(R, B)$, so $(a, F(a))$ is a soft lower bound of $(S, C)$ and hence $(a, F(a)) \leq_{s}\left(s^{*}, F\left(s^{*}\right)\right)$. Thus we have

$$
(a, F(a)) \leq_{s}\left(s^{*}, F\left(s^{*}\right)\right) \leq_{s}(b, F(b)) .
$$

So $\left(s^{*}, F\left(s^{*}\right)\right) \tilde{\in}(R, B)$. Similarly, we can prove that the soft greatest lower bound of $(S, C)$ is in $(R, B)$. Thus any $(S, C) \tilde{\subseteq}(R, B)$ has a soft greatest lower bound and a soft least upper bound in $(R, B)$ and hence the soft interval $(R, B)$ is a soft complete lattice with respect to the order induced by $\leq_{s}$.

Now we shall state and prove our main result.

Theorem 2.2 Let $\left((F, A), \leq_{S}\right)$ be a nonempty soft complete lattice and $f:(F, A) \stackrel{\sim}{\rightarrow}(F, A)$ a soft order-preserving. Then the soft mapping $f$ has a soft fixed point in $(F, A)$. Moreover, the soft set $\left(F_{f}, A^{0}\right)$ has a soft least (soft greatest) fixed point and is a soft complete lattice.

Proof. Let

$$
\left(P, A^{\prime}\right)=\left\{(x, F(x)) \tilde{\epsilon}(F, A):(x, F(x)) \leq_{s} f((x, F(x)))\right\}
$$

where

$$
A^{\prime}=\left\{x \in A: x \leq_{p} x^{\prime} \text { where } f((x, F(x)))=\left(x^{\prime}, F\left(x^{\prime}\right)\right) \text { and }(x, F(x)) \tilde{\epsilon}(F, A)\right\} .
$$

Since $(F, A)$ is the soft complete lattice, $\left(P, A^{\prime}\right)$ has a soft least upper bound $\left(b^{*}, F\left(b^{*}\right)\right)$ (say) in $(F, A)$. We show that $\left(b^{*}, F\left(b^{*}\right)\right)$ is a fixed point of $f$. As $\left(b^{*}, F\left(b^{*}\right)\right)$ is a soft upper bound of $\left(P, A^{\prime}\right)$, for every $(x, F(x)) \tilde{\in}\left(P, A^{\prime}\right)$ we have $(x, F(x)) \leq_{S}\left(b^{*}, F\left(b^{*}\right)\right)$. Note that

$$
(x, F(x)) \leq_{s} f((x, F(x))) \leq_{s} f\left(\left(b^{*}, F\left(b^{*}\right)\right)\right) .
$$

Thus $(x, F(x)) \leq_{s} f\left(\left(b^{*}, F\left(b^{*}\right)\right)\right)$ and hence $f\left(\left(b^{*}, F\left(b^{*}\right)\right)\right)$ is a soft upper bound of $\left(P, A^{\prime}\right)$. As $\left(b^{*}, F\left(b^{*}\right)\right)$ is a soft least upper bound of $\left(P, A^{\prime}\right)$, it follows that $\left(b^{*}, F\left(b^{*}\right)\right) \leq_{s} f\left(\left(b^{*}, F\left(b^{*}\right)\right)\right)$. 
Thus, we have $\left(b^{*}, F\left(b^{*}\right)\right) \tilde{\in}\left(P, A^{\prime}\right)$. Since $f$ is a soft order-preserving mapping, by (4) we obtain that $f\left(\left(b^{*}, F\left(b^{*}\right)\right)\right) \tilde{\epsilon}\left(P, A^{\prime}\right)$ which further implies that $f\left(\left(b^{*}, F\left(b^{*}\right)\right)\right) \leq_{s}\left(b^{*}, F\left(b^{*}\right)\right)$. Indeed, $\left(b^{*}, F\left(b^{*}\right)\right)$ is a soft least upper bound of $\left(P, A^{\prime}\right)$. Hence, $f\left(\left(b^{*}, F\left(b^{*}\right)\right)\right)=\left(b^{*}, F\left(b^{*}\right)\right)$. Next, we shall prove that the soft set $\left(F_{f}, A^{0}\right)$ has a soft least (soft greatest) fixed point. As $\left(F_{f}, A^{0}\right) \tilde{\subseteq}\left(P, A^{\prime}\right)$ and $\left(b^{*}, F\left(b^{*}\right)\right)$ is a soft upper bound of $\left(P, A^{\prime}\right)$, it follows that $\left(b^{*}, F\left(b^{*}\right)\right)$ is a soft upper bound of $\left(F_{f}, A^{0}\right)$. Hence $\left(b^{*}, F\left(b^{*}\right)\right)$ is the soft largest fixed point of $f$. Similarly, we can show that the soft greatest lower bound $\left(a^{*}, F\left(a^{*}\right)\right)$ of

$$
\left(Q, A^{\prime \prime}\right)=\left\{(x, F(x)) \tilde{\in}(F, A): f((x, F(x))) \leq_{s}(x, F(x))\right\}
$$

is the soft smallest fixed point of $f$, where

$$
A^{\prime \prime}=\left\{x \in A: x^{\prime \prime} \leq_{p} x \text { where } f((x, F(x)))=\left(x^{\prime \prime}, F\left(x^{\prime \prime}\right)\right) \text { and }(x, F(x)) \tilde{\epsilon}(F, A)\right\} .
$$

Indeed $\left(F_{f}, A^{0}\right) \tilde{\subseteq}\left(Q, A^{\prime \prime}\right)$ and $\left(a^{*}, F\left(a^{*}\right)\right)$ is a soft lower bound of $\left(Q, A^{\prime \prime}\right)$, it follows that $\left(a^{*}, F\left(a^{*}\right)\right)$ is a soft lower bound of $\left(F_{f}, A^{0}\right)$ and hence $\left(a^{*}, F\left(a^{*}\right)\right)$ is the soft smallest fixed point of $f$. We now prove that $\left(F_{f}, A^{0}\right)$ is a soft complete lattice. Let $(S, D)$ be a nonempty soft subsets of $\left(F_{f}, A^{0}\right)$. That is, $(S, D) \tilde{\subseteq}\left(F_{f}, A^{0}\right) \tilde{\subseteq}(F, A)$. As $\left(b^{*}, F\left(b^{*}\right)\right)$ is a soft upper bound of $\left(F_{f}, A^{0}\right)$ in $(F, A)$ and $(S, D) \tilde{\simeq}\left(F_{f}, A^{0}\right),\left(b^{*}, F\left(b^{*}\right)\right)$ is a soft upper bound of $(S, D)$ in $(F, A)$. Let $\left(s^{*}, F\left(s^{*}\right)\right)$ be a soft least upper bound of $(S, D)$ in $(F, A)$. Then we have $\left(s^{*}, F\left(s^{*}\right)\right) \leq_{s}\left(b^{*}, F\left(b^{*}\right)\right)$. By Lemma 2.1, $(R, B)$ is soft complete lattice. We claim that $f((x, F(x))) \tilde{\epsilon}(R, B)$ for all $(x, F(x)) \tilde{\epsilon}(R, B)$. For this, let $(x, F(x)) \tilde{\epsilon}(R, B)$. Since $f$ is a soft order-preserving mapping and $\left(b^{*}, F\left(b^{*}\right)\right) \tilde{\epsilon}\left(F_{f}, A^{0}\right)$, by $(3)$ we have $f((x, F(x))) \leq_{s}$ $\left(b^{*}, F\left(b^{*}\right)\right)$. By $(3)$ we obtain that $\left(s^{*}, F\left(s^{*}\right)\right) \leq_{s}(x, F(x))$. Thus

$$
(s, F(s)) \leq_{s}\left(s^{*}, F\left(s^{*}\right)\right) \leq_{s}(x, F(x))
$$

for all $(s, F(s)) \tilde{\epsilon}(S, D)$. Note that $(s, F(s)) \leq_{s} f((x, F(x)))$. Indeed, $(s, F(s)) \tilde{\in}(S, D) \tilde{\subseteq}\left(F_{f}, A^{0}\right)$ and $f$ is a soft order-preserving. So $f((x, F(x)))$ is a soft upper bound of $(S, D)$. As $\left(s^{*}, F\left(s^{*}\right)\right)$ is a soft least upper bound of $(S, D)$ in $(F, A)$, we have $\left(s^{*}, F\left(s^{*}\right)\right) \leq_{s} f((x, F(x)))$. Therefore we obtain that $\left(s^{*}, F\left(s^{*}\right)\right) \leq_{s} f((x, F(x))) \leq_{s}\left(b^{*}, F\left(b^{*}\right)\right)$ and $f((x, F(x))) \tilde{\in}(R, B)$. Since $(R, B)$ is a soft complete lattice in $(F, A), f$ has a soft smallest fixed point $\left(c^{*}, F\left(c^{*}\right)\right)$ (say) in $(R, B)$. Hence $\left(c^{*}, F\left(c^{*}\right)\right)$ is soft smallest upper bound of $(S, D)$ in $\left(F_{f}, A^{0}\right)$. Now $\left(c^{*}, F\left(c^{*}\right)\right) \tilde{\epsilon}(R, B)$ implies that $\left(s^{*}, F\left(s^{*}\right)\right) \leq_{s}\left(c^{*}, F\left(c^{*}\right)\right) \leq_{s}\left(b^{*}, F\left(b^{*}\right)\right)$. Also, $\left(s^{*}, F\left(s^{*}\right)\right) \leq_{s}$ $\left(c^{*}, F\left(c^{*}\right)\right)$ gives that $\left(c^{*}, F\left(c^{*}\right)\right)$ is a soft upper bound of $(S, D)$ in $\left(F_{f}, A^{0}\right)$. Now $\left(c^{*}, F\left(c^{*}\right)\right) \leq_{s}$ $\left(b^{*}, F\left(b^{*}\right)\right)$ implies that $\left(c^{*}, F\left(c^{*}\right)\right)$ is a soft least upper bound of $(S, D)$ in $\left(F_{f}, A^{0}\right)$. Following similar arguments to those given above, $(S, D)$ has soft greatest lower bound $\left(d^{*}, F\left(d^{*}\right)\right)$ (say) in $\left(F_{f}, A^{0}\right)$. As this holds for all $(S, D) \subseteq \tilde{\subseteq}\left(F_{f}, A^{0}\right)$, thus $\left(\left(F_{f}, A^{0}\right), \leq_{s}\right)$ is a soft complete lattice.

Corollary 2.3 Let $\left((F, A), \leq_{s}\right)$ be a soft complete lattice and $f:(R, B) \stackrel{\sim}{\rightarrow}(R, B)$ a soft order-preserving mapping. Then $f$ has a soft fixed point.

Proof. By lemma 2.1, $(R, B)$ is a soft complete lattice and the result follows from Theorem 2.2 . 
Example 2.4 Let $U=\left\{h_{1}, h_{2}, h_{3}, h_{4}, h_{5}, h_{6}, h_{7}, h_{8}\right\}$ be a given universe and $E=\left\{e_{1}, e_{2}, e_{3}, e_{4}, e_{5}, e_{6}, e_{7}\right.$, a given set of parameters. Suppose that $A=\left\{e_{1}, e_{3}, e_{5}, e_{8}\right\}$. Soft set $(F, A)$ over $U$ is given by

$$
(F, A)=\left\{\left(e_{1}, F\left(e_{1}\right)\right),\left(e_{3}, F\left(e_{3}\right)\right),\left(e_{5}, F\left(e_{5}\right)\right),\left(e_{8}, F\left(e_{8}\right)\right)\right\}
$$

where

$$
\begin{aligned}
& F\left(e_{1}\right)=\left\{h_{2}, h_{6}\right\}, F\left(e_{3}\right)=\left\{h_{2}, h_{3}, h_{6}\right\}, F\left(e_{5}\right)=\left\{h_{1}, h_{2}, h_{3}, h_{6}, h_{7}\right\}, \text { and } \\
& F\left(e_{8}\right)=\left\{h_{1}, h_{2}, h_{3}, h_{5}, h_{6}, h_{7}, h_{8}\right\} .
\end{aligned}
$$

Define the partial order $\leq_{p}$ on the set $A$ of parameters by

$$
e_{i} \leq_{p} e_{j} \text { if and only if } i \leq j .
$$

Clearly, $\left((F, A), \leq_{S}\right)$ is a partially ordered soft set. Note that, the ingredient $\left(e_{1}, F\left(e_{1}\right)\right)$ is the soft minimal and also the soft least element of $(F, A)$, the ingredient $\left(e_{8}, F\left(e_{8}\right)\right)$ is the soft maximal and the soft greatest element of $(F, A)$. Since all the ingredients of the ordered soft set $\left((F, A), \leq_{s}\right)$ are comparable and every nonempty soft subsets of $(F, A)$ has a soft least and soft greatest element, $\left((F, A), \leq_{s}\right)$ is soft complete lattice. Define a soft relation $f:(F, A) \stackrel{\sim}{\rightarrow}(F, A)$ by

$$
f=\left\{\begin{array}{c}
\left(\left(e_{1}, e_{3}\right), F\left(e_{1}\right) \times F\left(e_{3}\right)\right),\left(\left(e_{2}, e_{3}\right), F\left(e_{3}\right) \times F\left(e_{3}\right)\right), \\
\left(\left(e_{5}, e_{8}\right), F\left(e_{5}\right) \times F\left(e_{8}\right)\right),\left(\left(e_{8}, e_{8}\right), F\left(e_{8}\right) \times F\left(e_{8}\right)\right)
\end{array}\right\} .
$$

That is,

$$
\begin{aligned}
& f\left(\left(e_{1}, F\left(e_{1}\right)\right)\right)=\left(e_{3}, F\left(e_{3}\right)\right), \quad f\left(\left(e_{3}, F\left(e_{3}\right)\right)\right)=\left(e_{3}, F\left(e_{3}\right)\right), \\
& f\left(\left(e_{5}, F\left(e_{5}\right)\right)\right)=\left(e_{8}, F\left(e_{8}\right), \quad f\left(\left(e_{8}, F\left(e_{8}\right)\right)\right)=\left(e_{8}, F\left(e_{8}\right) .\right.\right.
\end{aligned}
$$

Note that $f$ is an order-preserving soft mapping. Thus all the conditions of Theorem 2.2 are satisfied. Moreover, soft ingredients $\left(e_{3}, F\left(e_{3}\right)\right)$ and $\left(e_{8}, F\left(e_{8}\right)\right)$ are soft least and soft greatest fixed points of $f$, respectively. Also, the soft fixed point set $\left(F_{f}, A^{0}\right)=\left\{\left(e_{3}, F\left(e_{3}\right)\right),\left(e_{8}, F\left(e_{8}\right)\right)\right\}$ is a soft complete lattice.

The soft Knaster-Tarski fixed point theorem ensures the existence of a soft common fixed point for a commuting family of soft isotone mappings.

Theorem 2.5 Let $\left((F, A), \leq_{s}\right)$ be a nonempty soft complete lattice and $\mathcal{F}$ a commuting family of soft order-preserving mappings from $(F, A)$ into $(F, A)$. Then $\left(F_{\mathcal{F}}, A^{0}\right)$, the set of soft common fixed points of all soft mappings $f \in \mathcal{F}$ is a nonempty soft complete sublattice of $(F, A)$.

Proof. Let

$$
\begin{aligned}
\left(P, A^{\prime}\right) & =\left\{(x, F(x)) \tilde{\epsilon}(F, A):(x, F(x)) \leq_{s} f((x, F(x)))\right\} \text { and } \\
\left(Q, A^{\prime \prime}\right) & =\left\{(x, F(x)) \tilde{\epsilon}(F, A): f((x, F(x))) \leq_{s}(x, F(x))\right\}
\end{aligned}
$$


where

$A^{\prime}=\left\{x \in A: x \leq{ }_{p} x^{\prime}\right.$ where $f((x, F(x)))=\left(x^{\prime}, F\left(x^{\prime}\right)\right)$ for all $f \in \mathcal{F}$ and $\left.(x, F(x)) \tilde{\epsilon}(F, A)\right\}$

and

$A^{\prime \prime}=\left\{x \in A: x^{\prime \prime} \leq_{p} x\right.$ where $f((x, F(x)))=\left(x^{\prime \prime}, F\left(x^{\prime \prime}\right)\right)$ for all $f \in \mathcal{F}$ and $\left.(x, F(x)) \tilde{\in}(F, A)\right\}$.

Since $(F, A)$ is soft complete lattice, so $\left(P, A^{\prime}\right)$ has a soft least upper bound $(u, F(u))$ (say) in $(F, A)$. Thus $(x, F(x)) \leq_{s}(u, F(u))$ for each $(x, F(x)) \tilde{\epsilon}\left(P, A^{\prime}\right)$. As $f$ is a soft order-preserving, we obtain that $f((x, F(x))) \leq_{s} f((u, F(u)))$. Now $(x, F(x)) \tilde{\in}\left(P, A^{\prime}\right)$ implies that $(x, F(x)) \leq_{s} f((x, F(x)))$. So, $(x, F(x)) \leq_{s} f((x, F(x))) \leq_{s} f((u, F(u)))$ for all $(x, F(x)) \tilde{\in}\left(P, A^{\prime}\right)$. Thus $f((u, F(u)))$ is a soft upper bound for $\left(P, A^{\prime}\right)$ and hence $(u, F(u)) \leq_{s}$ $f((u, F(u)))$ for all $f \in \mathcal{F}$. Let $g$ be any given element in $\mathcal{F}$. Then, we have

$$
g((u, F(u))) \leq_{s} g(f((u, F(u))))=f(g((u, F(u))))
$$

for any $f \in \mathcal{F}$. Thus $g((u, F(u))) \tilde{\epsilon}\left(P, A^{\prime}\right)$ and hence $g((u, F(u))) \leq_{s}(u, F(u))$. Since $g$ is an arbitrary soft mapping in $\mathcal{F}$, we obtain that $f((u, F(u)))=(u, F(u))$. Therefore $\left(F_{\mathcal{F}}, A^{0}\right) \neq \emptyset$. Similar results hold for the set $\left(Q, A^{\prime \prime}\right)$. From Theorem 2.2, it follows that $\left(F_{\mathcal{F}}, A^{0}\right)$ is a nonempty soft complete sublattice of $(F, A)$.

Example 2.6 Let partially ordered soft set $\left((F, A), \leq_{S}\right)$ and the soft mapping $f$ be as given in Example 2.4. Define a soft relation $g:(F, A) \sim(F, A)$ by

$$
g=\left\{\begin{array}{c}
\left(\left(e_{1}, e_{1}\right), F\left(e_{1}\right) \times F\left(e_{1}\right)\right),\left(\left(e_{3}, e_{3}\right), F\left(e_{3}\right) \times F\left(e_{3}\right)\right), \\
\left(\left(e_{5}, e_{8}\right), F\left(e_{5}\right) \times F\left(e_{8}\right)\right),\left(\left(e_{8}, e_{8}\right), F\left(e_{8}\right) \times F\left(e_{8}\right)\right)
\end{array}\right\} .
$$

That is,

$$
\begin{array}{ll}
g\left(\left(e_{1}, F\left(e_{1}\right)\right)\right)=\left(e_{1}, F\left(e_{1}\right)\right), & g\left(\left(e_{3}, F\left(e_{3}\right)\right)\right)=\left(e_{3}, F\left(e_{3}\right)\right), \\
g\left(\left(e_{5}, F\left(e_{5}\right)\right)\right)=\left(e_{8}, F\left(e_{8}\right)\right), & g\left(\left(e_{8}, F\left(e_{8}\right)\right)\right)=\left(e_{8}, F\left(e_{8}\right)\right) .
\end{array}
$$

Note that $g$ is an order-preserving soft mapping. The soft mappings $f$ and $g$ are commutative. Thus, $f$ and $g$ satisfy all the conditions of Theorem 2.5. Moreover, $\left(e_{3}, F\left(e_{3}\right)\right)$ and $\left(e_{8}, F\left(e_{8}\right)\right)$ are soft common fixed points of $f$ and $g$ in $(F, A)$, and the soft fixed point set $\left(F_{\mathcal{F}}, A^{0}\right)=$ $\left\{\left(e_{3}, F\left(e_{3}\right)\right),\left(e_{8}, F\left(e_{8}\right)\right)\right\}$ is soft complete sublattice of $(F, A)$.

\section{Acknowledgements}

The authors are sincerely grateful to the editor and referees for their careful reading, thoughtful suggestions and critical remarks for improving the quality of this manuscript. The first author is thankful to the financial assistance of the University of Pretoria Maths Pilot Doctoral bursary, the National Research Foundation (NRF) and DST-NRF Centre of Excellence in Mathematical and Statistical Sciences (CoE-MaSS). 


\section{References}

[1] M. Abbas, G. Murtaza and S. Romaguera, Soft contraction theorem, J. Nonlinear Convex Anal. 16 (3) (2015), 423-435.

[2] M. Abbas, G. Murtaza and S. Romaguera, On the fixed point theory of soft metric spaces, Fixed Point Theory Appl. 1 (2016), 1-11.

[3] M. Abbas, B. Ali and S. Romaguera, On generalized soft equality and soft lattice structure, Filomat 28 (6) (2014), 1191-1203.

[4] K. V. Babitha and J. J. Sunil, Soft set relations and functions, comput. Math. Appl. 60 (7) (2010), 1840-1849.

[5] K. V. Babitha and J. J. Sunil, Transitive closures and ordering on soft sets, comput. Math. Appl. 62 (5) (2011), 2235-2239.

[6] Y. M Bershchanskii and M. V Meerov, The complementarity problem: theory and methods of solution, Autom. Remote Control 44 (6) (1983), 687-710.

[7] K. C Border, Fixed point theorems with applications to economics and game theory, Cambridge University Press, 1985.

[8] S. Dafermos, Traffic equilibria and variational inequalities, Transportation science 14 (1) (1980), 42-54.

[9] S. Dafermos, Sensitivity analysis in variational inequalities, Math. Oper. Res. 13 (3) (1988), 421-434.

[10] A. C. Davis, A characterization of complete lattice, Pacific J. Math. 5 (1995), 311-319.

[11] X. Ding, N. Huang, F. Xia, Q. Zhang and Q. H. Ansari, Theory and algorithms of variational inequality and equilibrium problems, and their applications, Abstr. Appl. Anal. (2014), Hindawi Publ. Corp., 2014.

[12] M. Irfan Ali, T. Mahmood, M. Muti, U. Rehman and M. Fahim Aslam, On lattice ordered soft sets, Applied Soft Computing 36 (2015), 499-505.

[13] B. Knaster, Un théorème sur les fonctions d'ensembles, Ann. Soc. Polon. Math. 6 (1928), 133-134.

[14] P. K. Maji, R. Biswas and A. R. Roy, soft set theory, Comput. Math. Appl. 45 (4) (2003), 555-562.

[15] D. A. Molodtsov, Soft set theory-first results. Comput. Math. Appl. 37 (4) (1999), 19-31.

[16] A. Stouti, A fuzzy version of Tarski's fixpoint theorem, Arch. Math.(Brno) 40 (2004), 273-279. 
[17] D. Reem and S. Reich, Zone and double zone diagrams in abstract spaces, Colloquium Math. 115 (2009), 129-145.

[18] A. Tarski, A lattice-theoretical fixpoint theorem and its applications, Pacific J. Math. 5 (2) (1955), 285-309.

[19] D. Wardowski, On a soft mapping and its fixed points, Fixed Point Theory Appl. 1 (2013) (2013), 1-11.

[20] E. Zermelo, Neuer beweis für die möglichkeit einer wohlordnung, Math. Anal. 65 (1) (1907), 107-128. 